\title{
DRAMATURGIES CONTEMPORAINES: CROISADES, DE MICHEL AZAMA ET LE PROCESSUS DE TRADUCTION ${ }^{1 *}$
}

\author{
Márcio Bahia** \\ Maria Lúcia Jacob Dias de Barros ${ }^{* * *}$
}

Résumé: Dans la dramaturgie française contemporaine, l'cuvre de Michel Azama nous semble exemplaire en termes de modernité du point de vue des thèmes aussi bien que de l'écriture.

Nous présenterons ici certaines difficultés rencontrées dans la traduction de sa pièce "Croisades", dans le passage du français au portugais et les "solutions" proposées pour essayer de garder les particularités de cette écriture.

\section{L'ateur et L'ouvre}

Dans la dramaturgie française contemporaine, l'œuvre de Michel Azama nous semble exemplaire en termes de modernité aussi bien du point de vue des thèmes que de l'écriture.

\footnotetext{
* Texto entregue para publicação em novembro de 2004.

“ Graduado pela Faculdade de Letras/UFMG.

"* Professora da Faculdade de Letras/UFMG.

${ }^{1}$ Ce travail est lié à un projet universitaire ayant pour but diffuser la dramaturgie française contemporaine dans le milieu théatral et, si l'occasion se présente, au public en general à travers une future publication.
} 
Né en Catalogne en 1947, il a fait des études de Lettres Modernes à Montpellier puis a suivi une formation de comédien à l'école Jacques Lecoq et au Cours Simon à Paris. C'est là qu'il découvre ses dispositions pour l'écriture dramatique. Il a été successivement dramaturge au Nouveau Théâtre de Bourgogne, conseiller littéraire auprès du Centre National des Ecritures du Spectacle, rédacteur-en-chef de la revue Les cahiers de Prospéro. Il se consacre actuellement entièrement à l'écriture et conduit des ateliers d'écriture en France, Italie, Espagne, Colombie, Chili et traduit des auteurs espagnols, catalans et chiliens. Ces pièces ont été traduites en de nombreuses langues et représentées dans de nombreux pays d'Europe et d'Amérique Latine: Ruptures (1981), Géranium (1982), Bled (1983), Vie et mort de Pier Paolo Pasolini (1984), Le sas (1986), Croisades (1988), Iphigénie ou le pêché des dieux (1991), Aztèques (1992), et Amours fous (1993). ${ }^{2}$

Croisades, la pièce qui fait l'objet de notre étude, a déjà été traduite en douze langues et représentée dans plus de quinze pays, mais pas au Brésil. Dans cette pièce, AZAMA prend comme source d'inspiration un thème d'actualité - les guerres ethniques et religieuses - tout en faisant référence, par le titre même de la pièce, à un fait historique - les "croisades". C'est donc déjà le titre qui nous permet de transposer ces "croisades" du passé à la réalité d'aujourd'hui.

La pièce est composée d'un prologue et de quinze séquences où dix-sept personnages se croisent et dont les histoires se passent quelque part en Syrie ou bien en Israël, peut-être en Palestine, à Beyrouth ou Baalbec... Ces histoires sont toutes reliées à des guerres dont l'origine n'est pas précisée mais qui se perd dans le temps.

Dès le début de la pièce, dans le prologue, nous sommes devant deux petits enfants, un garçon et une fille, qui jouent à

\footnotetext{
${ }^{2}$ www.atelier-n89.com/html/azama.htm
} 
la guerre. En se servant de poupées, ils les manipulent en reproduisant toute l'horreur d'une vraie guerre. Les poupées, comme les êtres humains, sont brûlées, ont leurs membres du corps arrachés, les uns après les autres: les bras, les jambes et même la tête.

Dans les séquences qui suivent, nous découvrons d'autres personnages, de jeunes adultes cette fois, appartenant aux mêmes "tribus" mais qui, en conséquence de la guerre, deviennent des ennemis, se retrouvent dans des camps opposés et s'entretuent. Pendant cette guerre en permanence mais à des époques différentes, surgissent d'autres personnages plus âgés dont le rôle est d'accueillir les jeunes gens morts dans la guerre. Et finalement, une vieille dame, appelée Maman-Poule qui, apparemment, traverse toutes les époques, en direction de la Terre Promise, toujours à la recherche de ses enfants qui, eux, sont devenus des victimes de la guerre.

D'autres histoires et d'autres personnages se croisent également pour rendre encore plus vivant ce tableau aux airs d'apocalypse. Le passé et le présent se retrouvent par l'intermédiaire des personnages qui, même après leư mort, continuent à exister et nous livrent leur dernier message. L'auteur ajoute à l'horreur qui se déroule devant nous un certain humour et un sentiment de tendresse, une façon d'atténuer l'effet que pourraient causer les démonstrations de pure bestialité de la guerre.

\section{La traduction des textes littéraires}

Avant d'aborder notre travail de traduction proprement dit, nous voudrions présenter quelques réflexions à propos du processus de la traduction car il s'agit d'un sujet polémique qui se prête à discussion. 
Tout d'abord il y a ceux qui pratiquent la traduction (en tant que professionnels ou non), sans se soucier de réfléchir théoriquement sur leur travail; d'autres qui théorisent sur le processus de la traduction et finalement il y a aussi ceux qui sont en même temps traducteurs et théoriciens. Nous nous plaçons dans une autre catégorie: en tant que professeurs de langues étrangères, nous ne sommes pas traducteurs ni chercheurs dans ce domaine. Notre but ici sera, entre autres, de contribuer à la diffusion d'un auteur et d'une pièce de la dramaturgie française contemporaine, tout en relevant certains aspects qui ont trait au travail du traducteur.

Le point central des discussions concernant le travail du traducteur, c'est-à-dire, les rapports entre le texte original et le texte traduit, se rapporte à la question de la fidélité: Traduttore traditore? Le traducteur est-il un traitre?

D'un côté, il y a ceux qui croient que le texte traduit doit rester le plus proche possible du texte original. C'est le cas de José Paulo Paes qui, poète et traducteur, présente aussi ses réflexions sur ce type de travail:

"Verter um poema do grego, por exemplo, ou de qualquer outro idioma, é, teoricamente pelo menos, reescrevê-lo em português como o faria seu próprio autor, se tivesse domínio operativo de nossa língua, mas sem, no entanto, deixar de ser grego. Sublinho a última frase para destacar um ponto que reputo de capital importância. A idéia corrente de que boa é a tradução que dá ao leitor a mesma impressão de um texto originariamente escrito em sua língua pátria, constitui a maior das falácias." (PAES, 1990:93).

Par la suite, Paes nous éclaire un peu plus sur ce processus de transposition d'une langue à l'autre: il ne s'agit pas d'une 
opération d'isomorphie - une symétrie parfaite dans la forme et dans l'esprit - mais plutôt d'une opération de paramorphie- une similutude des formes et des sens. D'après lui, une symétrie serait impossible puisqu'on doit faire les adaptations nécessaires afin de respecter les particularités de la langue/culture en question, tout en gardant une certaine fidélité au texte original. Bien que ces adaptations se fassent nécessaires, Paes tient la fidélité comme le principe majeur de toute traduction.

D'un autre côté, Haroldo de Campos, poète et traducteur, défend l'idée selon laquelle le texte traduit peut être une recréation ou une création parallèle au texte original. Voici les termes de cette proposition déclarée d'une "subversion" du texte original:

"Numa tradução dessa natureza [tradução de textos criativos], não se traduz apenas o significado, traduz-se o próprio signo, ou seja, sua fisicalidade, sua materialidade mesma (propriedades sonoras, de imagética visual, enfim tudo aquilo que forma, segundo Charles Morris, a iconicidade do signo estético, entendido por signo estético aquele "que é de certa maneira similar àquilo que ele denota"). O significado, o parâmetro semântico, será apenas e tão-somente a baliza demarcatória do lugar da empresa recriadora. Está-se pois no avesso da chamada tradução literal" (DE CAMPOS, 1970:24).

La mission que s'est donnée le poète/traducteur de Campos mérite tout notre respect et admiration. Toutefois, en ce qui nous concerne, notre démarche s'insère dans les principes et les formulations de José Paulo Paes, c'est-à-dire, nous avons tenu à garder, autant que possible, toutes les significations du texte original. Notre souci était moins de garder la forme linguistique du texte à traduire que de trouver, 
dans le texte produit, une forme adéquate, en nous servant de toutes les ressources dont dispose la langue portugaise du Brésil, pour essayer de garder les caractéristiques d'un texte littéraire. Nous avions toujours à l'esprit, pour nous guider, la démarche de création d'un auteur comme Flaubert, qui, au moment où il écrivait ses romans, lisait chaque phrase à haute voix, pour essayer de lui donner les sonorités, le rythme, la mesure qu'il fallait pour la rendre belle, agréable à entendre, musicale même, comme s'il était en train d'écrire un poème.

Cette démarche utilisée nous a semblé d'autant plus nécessaire qu'il s'agit d'un texte dramatique dont le but, a priori, est d'être joué sur scène, n'étant donc pas l'objet d'une lecture silencieuse de la part d'un lecteur de romans ou d'un autre genre littéraire.

Il serait intéressant d'aborder ici certaines différences qui nous semblent exister, dans le processus de traduction, entre un texte littéraire dramatique et un texte littéraire non-dramatique. Ces aspects spécifiques et propres au texte dramatique, qui doivent être tenus en compte lors d'une traduction, sont abordés par Susan Bassnet. Selon cette essayiste anglaise, il existe très peu d'études concernant la traduction des textes dramatiques. D'autre part, si l'on se reporte aux remarques faites par les traducteurs eux-mêmes dont le nombre est assez peu significatif, cela nous mène à croire que le processus de traduction d'un texte dramatique est le même utilisé dans celle d'un texte nondramatique. Bassnet refuse cette idée car, selon cet auteur:

"Même si l'on considère cette question de façon superficielle, il est évident que le texte dramatique ne peut pas être traduit comme le serait un texte en prose. Tout d'abord, un texte dramatique est lu différemment . Il est lu comme quelque chose d'incomplet et non pas comme une unité achevée puisque ce n'est que dans sa 
performance [le jeu sur scène] que son potentiel est atteint complètement. Cela présente au traducteur: une problématique centrale: traduire le texte comme un texte purement littéraire ou essayer de le traduire en prenant en compte sa fonction en tant qu'élément faisant partie d'un autre système plus complexe." (BASSNET, 1980: 120).

Cet auteur semble convaincue que la démarche la plus adéquate serait celle qui prendrait en compte les études de sémiotique théâtrale selon lesquelles le texte dramatique n'est qu'une des composantes de cet ensemble de systèmes reliés dont se constitue un spectacle.

C'est pourquoi, étant du même avis, nous avons pris en compte, dans notre travail, ce caractère "virtuel" du texte dramatique, en le considérant, non pas comme un produit final, mais plutôt comme une des étapes de ce parcours: texte original $\rightarrow$ texte traduit $\rightarrow$ mise-en-scène.

\section{D'un texte à l'autre}

Comme nous l'avons fait remarquer ci-dessus, notre souci, dans le processus de traduction, était de garder, autant que possible, la plus grande fidélité au texte original. Néanmoins, nous avons été confrontés à certaines difficultés qui, pour être résolues, nous ont conduits à faire certains choix. Pour les rendre plus clairs dans cette présentation, nous avons donc établi quelques points de repère:

1. En ce qui concerne les noms propres, nous les avons respectés en gardant les mêmes noms des personnages et des lieux, notre intention n'étant pas d'adapter la réalité décrite dans la pièce à une réalité brésilienne. Par exemple, au lieu d'Ismael et Jonatan, nous avons préféré maintenir Ismaill et Yonathan, de même que 
Krim, Bella et Zack. Quant aux autres personnages, qui n'avaient pas de noms propres, ils ont simplement été traduits: "la petite fille" = "a menina", "l'indien Peau Rouge" = "o índio Pele Vermelha", etc. Pour ce qui est du personnage de Maman Poule, nous avons opté pour lui donner le nom de "Mãe Coruja" puisque la traduction littérale, "Mamãe Galinha" Iui attribuerait des caractéristiques qui ne correspondaient pas à celles du personnage. Au Brésil, en parlant d'une femme, "galinha" est un mot péjoratif et exprime une conduite sexuelle de promiscuité. Par contre, "Mãe Coruja" exprime bien l'idée d'une mère qui se soucie de ses enfants, qui cherche tout le temps à les protéger, correspondant exactement aux traits psychologiques du personnage.

2. Par rapport à la ponctuation, l'absence de virgules et l'utilisation uniquement des points et des points d'interrogation a été maintenue. Comme il s'agit d'un texte qui va être joué sur scène, c'est à l'acteur de lui conférer le rythme, l'intonation et les pauses que requiert le langage parlé, en forme de dialogue. Exemple: "D'ailleurs moi le silence ça m'angoisse" = "Além do mais o silêncio me deixa angustiado".

3. Le caractère d'oralité, manifeste dans les dialogues, a constitué le souci majeur dans notre travail et pour cela nous avons essayé de garder les caractéristiques de la langue parlée par les personnages. Pourtant, quand il s'agissait des répliques énoncées par les jeunes gens, qui se servent d'un vocabulaire propre au langage familier, la plupart du temps nous n'avons pas pu utiliser le même registre de langue qu'en français: "Vous me foutez la trouille" n'est pas dans le même 
registre que "Vocês estão me matando de medo"; idem pour "Fais gaffe" = "Tome cuidado". Nous avons eu le même problème au niveau des structures grammaticales, par exemple: "La guerre il y a rien de plus génial même les filles ça peut pas être plus génial" = "Não tem nada mais legal que a guerra nem mesmo as mulheres".

Une autre remarque importante à faire concernant la langue parlée est la disparition très fréquente, en français, de la particule négative ne. En portugais nous n'avons pas cette marque d'oralité, d'où l'impossibilité de la reproduire dans notre texte. Par contre, il y en a d'autres qui n'existent pas en français. Nous avons choisi de maintenir, dans quelques cas, certaines de ces marques d'oralité en portugais et les avons évitées dans d'autres. En ce qui concerne la place du pronom personnel objet, nous l'avons maintenue dans les exemples:

a) "C'est facile. Embrasse-moi" = "É fácil. Me beije";

b) "Il m'en veut de l'avoir tué" = Ele está com raiva de mim por ter matado elle".

Dans d'autres exemples, au contraire, nous avons évité ces marques:

c) "J'essaie de ressouder des morceaux de mon visage. Je le masse. Il a l'air intact." = "Tento juntar os pedaços do meu rosto. Fico massageando meu rosto. Ele parece intacto" (l'alternative "Fico massageando elle" a été évitée pour des questions d'euphonie).

Une autre marque d'oralité du portugais, la possibilité d'ommettre le pronom sujet en début de phrase, a été utilisée selon les nécessités de la structure de la langue portugaise elle-même:

a) "J'ai peur de tomber" = "Estou com medo de cair";

b) "J'étais là. J'ai tout vu" = "Eu estava lá. (Eu) vi tudo".

4. Dans le but de rendre plus clairs les processus de cette 
"transposition" d'une langue à l'autre, nous avons pris comme modèle de référence les modalités de la traduction "oblique" établies par Gerardo VÁZQUEZAYORA (1977)3, dont le travail nous a été présenté par José Luiz VILA REAL GONÇALVES dans Teorizando $e$ contextualizando a tradução. (VIEIRA, org., 1996)

VAZQUEZ-AYORA définit deux types de traduction: l'une, littérale, où nous retrouvons un parallélisme lexical et syntaxique et la possibilité de quelques ajustements minimes ("normalisation syntaxique"); l'autre, par opposition à celle-là, où ce parallélisme n'est pas présent, qu'il appelle traduction "oblique". Ces concepts de traduction "littérale" et "oblique" sont à rapprocher de ceux présentés par José Paulo PAES, isomorphie et paramorpbie, cités plus haut.

Dans la traduction "oblique", nous retrouvons les modalités suivantes, repérées dans notre travail et dont nous présentons la définition ${ }^{4}$, suivies de quelques exemples:

a) Transposition - "La transposition est un procédé qui permet l'alternance stylistique, pouvant être obligatoire ou facultative. C'est l'un des procédés les plus récurrents et les plus importants dans la traduction". Nous avons pris en exemple la phrase "Elle a l'habitude de me voir vivant" = "Ela está acostumada a me ver vivo".

b) Modulation - "changement de point de vue, qui se justifie quand la traduction littérale modifie le sens de la langue de réception." Exemple: "Le ne suis pas dans le coup" = "Estou fora".

c) Equivalence - "considérée comme un cas extrême de

${ }^{3}$ Introducción a la tradutología - Curso básico de traducción. Georgetown: Georgetown University, 1977.

`Ces définitions ont été traduites, du portugais au français, par nous-mêmes. 
modulation. (...) Elle cherche la vision exocentrique et l'effet total". Exemples:

"LE PETIT VIEUX

Vous venez de casser votre pipe.

LA PETITE VIEILLE

De lâcher la rampe de dévisser votre billard de fermer votre parapluie...

LE PETIT VIEUX

De rendre votre clé de mettre la table pour les asticots...

LA PETITE VIEILLE

D'avaler votre bulletin de naissance."

Dans notre traduction:

"O VELHINHO

Você acabou de bater as botas.

A VELHINHA

Abotoar o paletó esticar a canela bater a caçoleta.

O VELHINHO

Ir desta pra melhor bater o trinta e um.

A VELHINHA

De virar presunto."

d) Adaptation - "adéquation d'un contenu aux particularités culturelles de la langue d'origine." Exemple: "Si naturel d'être abattus sur la terrasse pendant une partie de boules" = "Tão natural ser abatido no terraço durante um jogo de damas."

e) Amplification - "(...) quand un terme ou une expression de la langue d'origine a plus de densité sémantique que son équivalent" dans la langue de réception. Exemple: 


\section{"ISMAIL}

Il n'y a pas un seul sentiment dans toute ta carcasse. YONATHAN

Si."

Dans notre traduction:

"ISMAIL

Não existe nem um único sentimento em toda essa sua carcaça.

YONATHAN

Existe sim"

f) Explicitation - "procédé d'explicitation de ce qui est implicite dans la langue d'origine."Exemple:

"KRIM

C'est ça qui te trompe. C'est des idées. Faut pas se fier à des idées. Ils nous infiltrent."

Dans notre traduction:

"KRIM

É aí que você se engana. É com essas idéias. Não dá pra confiar nas idéias. É assim que eles se infiltram na gente."

g) Ommission - "opération contraire à l'explicitation, c'est'a-dire élimination de ce qui est redondant." Exemple: "Un seau plein de grenades ça fait mal." = "Um balde cheio de granadas machuca."

Nous devons souligner que, malgré les distinctions faites entre les différentes modalités, il se peut qu'il y ait plus d'une modalité dans un seul cas.

5. Quand nous traduisons un texte ou une œuvre d'une langue à l'autre, nous faisons nécessairement une transposition d'une culture à l'autre. Cet aspect interculturel est donc intrinsèque à tout travail de traduction. Cependant, dans un pays aux dimensions 
continentales comme le nôtre, en plus de cette interculturalité, il faut relever aussi un autre aspect important: le caractère "intraculturel", c'est-à-dire, les spécificités, les régionalismes propres à chaque région. Dans notre cas, il s'agit d'une situation assez particulière puisque nous avons réalisé ce travail à quatre mains, deux personnes originaires de régions très différentes, l'une venant du Nord du pays, l'autre du Sud-est du Brésil (plus précisément, du Minas). Nous avons donc préféré utiliser une langue "standard" en ce qui concerne le vocabulaire ainsi que les pronoms personnels sujet ("você" au lieu de "tu" ou "cê").

Pour illustrer cette démarche, voici un exemple des choix que nous avions et de celui que nous avons fait:

"T'es bête" pourrait avoir comme équivalent "Tu és um abestado" (comme diraient les gens du Nord) ou "Égua. do pequeno abestado" (particulièrement dans le Pará) ou encore "Cê é besta" (dans le Minas) mais la forme choisie a été "Deixa de ser bobo", qui ne semblerait pas bizarre aux oreilles d'un Brésilien quelle que soit sa région natale.

Pour conclure, nous espérons que ces réflexions concernant le processus de traduction pourront contribuer à la réalisation d'autres travaux de traduction et de recherche et que, du point de vue de l'enseignement/apprentissage d'une langue étrangère, elles pourront profiter également aux professeurs de langue et de littérature françaises. 
Resumo: Na dramaturgia contemporânea francesa, a obra de Michel Azama nos parece exemplar em termos de modernidade tanto do ponto de vista temático quanto da escritura.

Apresentaremos aqui certas dificuldades encontradas na tradução de sua peça "Croisades", na passagem do francês para o português e as "soluções" propostas para tentar conservar as particularidades dessa escritura.

\section{Références bibliographiques}

AZAMA, Michel, Croisades. Paris: Editions Théatrales, 1992.

AZAMA, Michel, Zoo da noite/A cela. Trad. Edson Rodrigues, Rio de Janeiro: Ed. 7 Letras, 2000.

BASSNETT-MCGUIRE, Susan, Translation Studiesnew (new accents). Methuen, London and New York, 1980.

CAMPOS, Haroldo de, "Da tradução como criação e como crítica".In: Metalinguagem. Sāo Paulo: Cultrix, 1970, p.21-38.

GONÇALVES, José Luiz, "VÁSQUEZ-AYORA, Gerardo, Introducción a la tradutología- Curso básico de traducción". In: VIEIRA, EIse Ribeiro Pires(org.), Teorizando e contextualizando a tradução. Belo Horizonte: Faculdade de Letras da UFMG, Cours de "Pós-graduação em estudos lingüísticos", 1996, p. 59-67.

PAES, José Paulo, Tradução: a ponte necessária. São Paulo: Ática, 1990.

SITE

www.atelier-n89.com/html/azama.htm 